\title{
AN ALTERNATIVE MODEL FOR THE DUFFIN-KEMMER-PETIAU OSCILLATOR
}

\author{
D. A. Kulikov, R. S. Tutik* and A. P. Yaroshenko, \\ Dniepropetrovsk National University, \\ Kazakova Str. 20, Dniepropetrovsk, 49050, Ukraine
}

March 1, 2018

\begin{abstract}
A new oscillator model with different form of the nonminimal substitution within the framework of the DuffinKemmer-Petiau equation is offered. The model possesses exact solutions and a discrete spectrum of high degeneracy. The distinctive property of the proposed model is the lack of the spin-orbit interaction, being typical for other relativistic models with the non-minimal substitution, and the different value of the zero-point energy in comparison with that for the Duffin-Kemmer-Petiau oscillator described in the literature.
\end{abstract}

Keywords: Duffin-Kemmer-Petiau equation; bound states; oscillator model.

PACS Nos. 03.65.Ge, 03.65.Pm

*tutik@ff.dsu.dp.ua 


\section{Introduction}

The harmonic oscillator is one of the most basic and useful solvable examples in the non-relativistic quantum mechanics. Recently, the harmonic-oscillator potential for relativistic spin-1/2 particles has received considerable attention by many groups. Besides the academic importance of the exact solutions, the relativistic oscillator may be an analytical basis to deal with more realistic interactions in several areas, namely, in nuclear and particle physics. In addition to the exact solutions of the Dirac equation with confining scalar-vector potentials 1, 2, 3, 4, since the paper by Moshinsky and Szczepaniak [5], the interest has been extended to the oscillator model constructed by means of a non-minimal coupling scheme.

This model, introduced formerly in the works 6, 7, 8, 9, has the Hamiltonian which is linear in both momenta and coordinates, conserves angular momentum, is exactly solvable and its eigenspectrum is highly degenerate. The system obtained, being a harmonic oscillator with a spin-orbit coupling in the non-relativistic limit, is referred to as the Dirac oscillator (for further references see Ref. 10).

Analogous models have been constructed within the framework of the KleinGordon equation [11, 12], and the spin-0 and spin-1 Duffin-Kemmer-Petiau (DKP) formalism, too. In the latter case, there are two different approaches. The first approach [13, 14, the PSUSY oscillator, employs ideas of the parasupersymmetric quantum mechanics [15] whereas the second one [16], the DKP oscillator, exploits the technique developed for the Dirac oscillator [5]. However, the PSUSY oscillator has been proved to be equivalent to the DKP oscillator supplemented with the additional constraint 17 .

The eigenstates of the spin-1 DKP oscillator subdivide into the states with the natural and unnatural parities, whereas for the PSUSY oscillator only the naturalparity states become permissible due to the above-mentioned constraint. It should be pointed out that the energy eigenvalues of unnatural-parity states of the spin1 DKP oscillator show the behavior which is unusual for oscillator models. These energy eigenvalues are not equidistant in contrast to those of the spin- 0 and spin- $1 / 2$ oscillators and the spectrum of the spin-1 oscillator with the natural-parity states.

The goal of the present work is to study a more general type of the non-minimal substitution allowed within the framework of the DKP equation and to show that there is an alternative model of the spin- 1 DKP oscillator whose energy spectrum is equidistant for the states of both the natural and unnatural parity.

\section{Generalization of the Model of the Duffin-Kemmer- Petiau Oscillator}

In this section we sketch out the technique of the non-minimal substitution and consider the generalization of the model of the DKP oscillator.

Let us recall that the Duffin-Kemmer-Petiau equation [18, 19, 20, describing a free scalar or vector boson of the nonzero mass $m$ can be written as

$$
i \hbar \beta^{0} \frac{\partial \psi}{\partial t}=\left(c \vec{\beta} \cdot \vec{p}+m c^{2}\right) \psi,
$$

where the matrices $\beta^{\mu}(\mu=0,1,2,3)$ satisfy the Kemmer trilinear algebra

$$
\beta^{\mu} \beta^{\nu} \beta^{\lambda}+\beta^{\lambda} \beta^{\nu} \beta^{\mu}=g^{\mu \nu} \beta^{\lambda}+g^{\lambda \nu} \beta^{\mu}
$$

and the convention for the metric tensor is $g^{\mu \nu}=\operatorname{diag}(1,-1,-1,-1)$.

Actually, there exist different representations for this algebra [21]. However, the most used among them are the following three very well known cases: the trivial 
one with $\beta^{\mu}=0$, a five-dimensional representation describing the spin-0 particles, and a ten-dimensional one for the spin- 1 bosons. In the last case, the matrices $\beta^{\mu}$ in the notation of the paper [16] are given by

$$
\beta^{0}=\left(\begin{array}{cccc}
0 & \overline{0} & \overline{0} & \overline{0} \\
\overline{0}^{T} & \mathbf{0} & \mathbf{I} & \mathbf{0} \\
\overline{0}^{T} & \mathbf{I} & \mathbf{0} & \mathbf{0} \\
\overline{0}^{T} & \mathbf{0} & \mathbf{0} & \mathbf{0}
\end{array}\right), \quad \beta^{i}=\left(\begin{array}{cccc}
0 & \overline{0} & e_{i} & \overline{0} \\
\overline{0}^{T} & \mathbf{0} & \mathbf{0} & -i s_{i} \\
-e_{i}^{T} & \mathbf{0} & \mathbf{0} & \mathbf{0} \\
\overline{0}^{T} & -i s_{i} & \mathbf{0} & \mathbf{0}
\end{array}\right), \quad i=1,2,3
$$

where $s_{i}$ are the usual $3 \times 3$ spin- 1 matrices and

$$
\overline{0}=\left(\begin{array}{lll}
0 & 0 & 0
\end{array}\right) \quad e_{1}=\left(\begin{array}{lll}
1 & 0 & 0
\end{array}\right) \quad e_{2}=\left(\begin{array}{lll}
0 & 1 & 0
\end{array}\right) \quad e_{3}=\left(\begin{array}{lll}
0 & 0 & 1
\end{array}\right)
$$

while $\mathbf{0}$ and $\mathbf{I}$ stand for the $3 \times 3$ zero and identity matrices, respectively.

Inserting the non-minimal substitution

$$
\vec{p} \rightarrow \vec{p}-i m \omega \eta_{0} \vec{r}
$$

into (11) we obtain the equation for the DKP oscillator [16]

$$
i \hbar \beta^{0} \frac{\partial \psi}{\partial t}=\left[c \vec{\beta} \cdot\left(\vec{p}-i m \omega \eta_{0} \vec{r}\right)+m c^{2}\right] \psi
$$

where $\eta_{0}=2\left(\beta^{0}\right)^{2}-1$ and $\omega$ is the oscillator frequency.

We attempt to generalize this model through a modification of the non-minimal substitution. For this purpose we extend the coupling term to the more general form

$$
\vec{p} \rightarrow \vec{p}-i m \omega M \vec{r},
$$

where $M$ is some $10 \times 10$ matrix consisted of combinations of matrices $\beta^{\mu}$.

Obviously, not every matrix $M$ will lead us to an oscillator model. As it was noticed in Ref. 12, in models of both the spin-0 and spin- $1 / 2$ oscillators, a matrix involving a potential in the non-minimal substitution anticommutes with the matrix structure of the momentum part of the wave equation. We suppose that this property remains valid as well for a model of the spin-1 oscillator. In our case, the such matrix is $M$, so that the following condition must be held

$$
\vec{\beta} M+M \vec{\beta}=0 .
$$

For the Kemmer algebra (2), there exist four matrices anticommuting with $\vec{\beta}$. Hence, $M$ may be chosen as their linear combination

$$
M=a_{1} \eta_{0}+a_{2} \eta_{5}+a_{3} \eta+a_{4} \eta_{0} \eta_{5} \eta
$$

where $a_{i}$ are numerical coefficients and the matrices $\eta_{5}$ and $\eta$ are given by

$$
\begin{aligned}
\eta_{5} & =-\left(2\left(\beta^{0}\right)^{2}-1\right)\left(2\left(\beta^{1}\right)^{2}+1\right)\left(2\left(\beta^{2}\right)^{2}+1\right)\left(2\left(\beta^{3}\right)^{2}+1\right), \\
\eta & =2 \beta^{\mu} \beta_{\mu}-5
\end{aligned}
$$

Exploiting the spin-1 representation for $\beta^{\mu}$ we have

$$
\eta=-\eta_{5}, \quad \eta_{0} \eta_{5} \eta=-\eta_{0},
$$

and the final expression for the matrix $M$ reduces to

$$
M=b_{1} \eta_{0}+b_{2} \eta_{5} .
$$


Thus, the DKP equation with the non-minimal substitution (7) which includes the matrix $M$ in the form (12) is written as

$$
i \hbar \beta^{0} \frac{\partial \psi}{\partial t}=\left[c \vec{\beta} \cdot\left(\vec{p}-i m \omega\left(b_{1} \eta_{0}+b_{2} \eta_{5}\right) \vec{r}\right)+m c^{2}\right] \psi .
$$

Next, the block structure (3) of the matrices $\beta^{\mu}$ implies that the ten-component wave function of a stationary state may be decomposed into

$$
\psi(\vec{r}, t)=\left(\begin{array}{llll}
i \varphi(\vec{r}) & \vec{F}(\vec{r}) & \vec{G}(\vec{r}) & \vec{K}(\vec{r})
\end{array}\right)^{T} \exp (-i E t / \hbar)
$$

and the equation (13) gives rise to the following set of time-independent equations

$$
\begin{aligned}
m c^{2} \varphi & =i c\left(\vec{p}-i\left(b_{1}+b_{2}\right) m \omega \vec{r}\right) \cdot \vec{G}, \\
m c^{2} \vec{F} & =E \vec{G}-c\left(\vec{p}+i\left(b_{1}-b_{2}\right) m \omega \vec{r}\right) \times \vec{K}, \\
m c^{2} \vec{G} & =E \vec{F}+i c\left(\vec{p}+i\left(b_{1}+b_{2}\right) m \omega \vec{r}\right) \varphi, \\
m c^{2} \vec{K} & =-c\left(\vec{p}-i\left(b_{1}-b_{2}\right) m \omega \vec{r}\right) \times \vec{F} .
\end{aligned}
$$

Upon eliminating $\varphi(\vec{r}), \vec{G}(\vec{r})$ and $\vec{K}(\vec{r})$ this set becomes equivalent to the only one equation for the 3 -component spinor $\vec{F}(\vec{r})$

$$
\begin{aligned}
& \left(E^{2}-m^{2} c^{4}\right) \vec{F}=\left[c^{2}\left(\vec{p}^{2}+\left(b_{1}-b_{2}\right)^{2} m^{2} \omega^{2} \vec{r}^{2}\right)\right. \\
& \left.-\left(3 b_{1}-b_{2}\right) \hbar \omega m c^{2}-2 b_{1} \omega m c^{2} \vec{L} \cdot \vec{s}\right] \vec{F}+4 b_{1} b_{2} c^{2} m^{2} \omega^{2} \vec{r}(\vec{r} \cdot \vec{F}) \\
& -\frac{2 b_{1} \omega}{m}\left(\vec{p}+i\left(b_{1}+b_{2}\right) m \omega \vec{r}\right)\left[\left(\vec{p}-i\left(b_{1}-b_{2}\right) m \omega \vec{r}\right) \cdot(2 \hbar+\vec{L} \cdot \vec{s}) \vec{F}\right],
\end{aligned}
$$

where $\vec{L}$ is the orbital angular momentum and $\vec{s}$ is the $3 \times 3$ spin- 1 operator.

The derived equation, apart from the last term, is that for a harmonic oscillator. However, as $c \rightarrow \infty$ this last term becomes negligible and in the non-relativistic limit the described system corresponds to the ordinary harmonic oscillator of frequency $\omega$ with a strong spin-orbit coupling term. This justifies the name of the DuffinKemmer-Petiau oscillator for our model as well.

\section{Exact solutions to the derived equation}

Because within the framework of the DKP equation the total angular momentum $\vec{J}=\vec{L}+\hbar \vec{s}$ is conserved, the spatial variables for the components of the wave function (14) are separated and we can write 22]

$$
\begin{array}{lll}
\varphi=\frac{1}{r} \phi(r) Y_{J M}(\Omega), & \vec{F}=\frac{1}{r} \sum_{L} f_{L}(r) \vec{Y}_{J L 1}^{M}(\Omega), \\
\vec{G}=\frac{1}{r} \sum_{L} g_{L}(r) \vec{Y}_{J L 1}^{M}(\Omega), & \vec{K} & =\frac{1}{r} \sum_{L} k_{L}(r) \vec{Y}_{J L 1}^{M}(\Omega),
\end{array}
$$

where $\vec{Y}_{J L 1}^{M}(\Omega)$ are the so-called vector spherical harmonics.

Substituting (17) into (15) leads to ten radial equations which reduce to the two uncoupled sets associated with the $(-1)^{J}$ and $(-1)^{J+1}$ parities. The $(-1)^{J}$ solutions correspond to the natural-parity states. They are described by the functions (17) with the nonzero components $f_{J}, g_{J}, k_{J \pm 1}$. The orbital angular momentum for these states has a definite value $J=L$. The $(-1)^{J+1}$ solutions, with values of $J=L-1$ and $J=L+1$ being mixed, are referred to as the unnatural-parity states. Their nonzero components are $f_{J \pm 1}, g_{J \pm 1}, k_{J}, \phi$. 
In the case of the natural-parity states, the radial equation has the oscillator-like form

$$
\begin{aligned}
& \left(\hbar^{2} \frac{\mathrm{d}^{2}}{\mathrm{~d} r^{2}}+\frac{E^{2}-m^{2} c^{4}}{c^{2}}+\left(b_{1}-b_{2}\right) \hbar \omega m\right. \\
& \left.-\left(b_{1}+b_{2}\right)^{2} m^{2} \omega^{2} r^{2}-\frac{\hbar^{2} J(J+1)}{r^{2}}\right) f_{J}(r)=0,
\end{aligned}
$$

whereas for the unnatural-parity states one gets a system of two coupled equations

$$
\begin{aligned}
\left(\hbar^{2} \frac{\mathrm{d}^{2}}{\mathrm{~d} r^{2}}\right. & \left.+\frac{E^{2}-m^{2} c^{4}}{c^{2}}-3\left(b_{1}+b_{2}\right) \hbar \omega m-\left(b_{1}+b_{2}\right)^{2} m^{2} \omega^{2} r^{2}-\frac{\hbar^{2} J(J+1)}{r^{2}}\right) \phi(r) \\
& -\frac{2 b_{1} \sqrt{J(J+1)} \hbar \omega E}{c^{2}} k_{J}(r)=0, \\
\left(\hbar^{2} \frac{\mathrm{d}^{2}}{\mathrm{~d} r^{2}}\right. & \left.+\frac{E^{2}-m^{2} c^{4}}{c^{2}}-\left(b_{1}-b_{2}\right) \hbar \omega m-\left(b_{1}-b_{2}\right)^{2} m^{2} \omega^{2} r^{2}-\frac{\hbar^{2} J(J+1)}{r^{2}}\right) k_{J}(r) \\
& -\frac{2 b_{1} \sqrt{J(J+1)} \hbar \omega E}{c^{2}} \phi(r)=0 .
\end{aligned}
$$

Now we consider exact solutions to the last equations. It is easily seen that the obtained system decouples into two oscillator-like equations and, hence, has exact solutions if and only if $b_{1}=0$ or $b_{2}=0$. For these two cases we have:

(i) $b_{1}=1, \quad b_{2}=0$ (the value $b_{1}=$ const $\neq 1$ can be absorbed into $\omega$ providing $\left.b_{1}=1\right)$. This choice of coefficients reproduces the DKP oscillator model proposed in Ref. 16. According to (18) and (19), its eigenvalues take the form

$$
\begin{aligned}
E^{2}-m^{2} c^{4} & =(4 n+2 J+2) \hbar \omega m c^{2}(L=J), \\
E^{2}-m^{2} c^{4}+\hbar \omega \sqrt{m^{2} c^{4}+4 J(J+1) E^{2}} & =(4 n+2 J+3) \hbar \omega m c^{2}(L=J \pm 1), \\
E^{2}-m^{2} c^{4}-\hbar \omega \sqrt{m^{2} c^{4}+4 J(J+1) E^{2}} & =(4 n+2 J+3) \hbar \omega m c^{2}(L=J \pm 1(20)
\end{aligned}
$$

where $n$ is the radial quantum number. Here the first equation describes the energies of the natural-parity states and the rest equations correspond to the unnaturalparity states.

(ii) $b_{1}=0, \quad b_{2}=1$. With these coefficients we arrive at the alternative model for the DKP oscillator. Its eigenvalues are given by

$$
\begin{aligned}
& E^{2}-m^{2} c^{4}=(4 n+2 J+4) \hbar \omega m c^{2}(L=J), \\
& E^{2}-m^{2} c^{4}=(4 n+2 J+6) \hbar \omega m c^{2}(L=J \pm 1), \\
& E^{2}-m^{2} c^{4}=(4 n+2 J+2) \hbar \omega m c^{2}(L=J \pm 1)
\end{aligned}
$$

that can be rewritten with the common formula

$$
E^{2}-m^{2} c^{4}=(4 n+2 L+4) \hbar \omega m c^{2} \quad(L=J-1, J, J+1) .
$$

In contrast to the equations (20), the eigenspectrum of this model proved to be completely equidistant. Besides, these models differ in the zero-point energy.

Note, that in the case $J=0$, the radial equations should be deduced individually with putting $f_{-1}(r)=g_{-1}(r)=k_{-1}(r)=0$ into (17). For all this the expression (22) for the eigenvalues with $L=J+1=1$ remains still valid.

\section{Discussion}

Thus, we have considered the DKP equation with the interaction introduced through the non-minimal substitution of general form. This allows us to find a new exactly 
solvable model for the spin-1 oscillator that possesses the completely equidistant eigenspectrum.

Moreover, the eigenvalues of the proposed model differ from those of the spin-0 oscillator [11, 12] in appearance only the constant addition term $4 \hbar \omega$. It means that within the framework of the alternative model the spin-1 particle behaves like the spinless one. In fact, as soon as we insert $b_{1}=0$ into (16) the terms with the spin-orbit coupling vanish there.

Hence, it appears that the exactly solvable models for the DKP oscillator considered in the previous section differ dramatically from each other. This should be regarded as arising from the different Lorentz structure of the oscillator coupling terms in these models.

For elucidating this difference let us turn to the Lorentz covariant form of the DKP equation (13)

$$
\left[c \beta^{\mu} p_{\mu}+\frac{1}{2} b_{1}\left(S_{\mu \nu} \beta_{\lambda}+\beta_{\lambda} S_{\mu \nu}\right) T^{\mu \nu \lambda}-i b_{2} \beta_{\mu} \eta_{5} V^{\mu}-m c^{2}\right] \psi=0,
$$

where $S_{\mu \nu}=i\left(\beta_{\mu} \beta_{\nu}-\beta_{\nu} \beta_{\mu}\right)$ is the tensor of spin, and external potentials are involved in the Lorentz-tensor, $T^{\mu \nu \lambda}$, and Lorentz-vector, $V^{\mu}$, interactions in spinor space. Then the equation (13) is immediately restored with setting

$$
V^{0}=T^{000}=T^{i j 0}=T^{\mu \nu i}=0, \quad V^{i}=T^{0 i 0}=-T^{i 00}=m \omega x^{i} .
$$

Taking into account (23), we conclude that the model of the DKP oscillator with $b_{1}=1, \quad b_{2}=0$ considered in the paper [16 realizes the case of the Lorentztensor coupling, whereas the alternative model with $b_{1}=0, \quad b_{2}=1$ proposed here includes the coupling of the Lorentz-vector type.

A few remarks concerning the lack of the spin-orbit coupling are in order. There is another way to introduce the harmonic oscillator in relativistic quantum mechanics. It has been realized within the framework of the Dirac equation by means of mixing vector and scalar harmonic potentials with equal magnitude and equal or opposite sign (for references see Ref. 10), that results in a quadratic equation for each spinor component. The energy eigenvalues obtained in the such approach are characterized by the $(2 n+l)$ degeneracy and the lack of contribution from the spin-orbit interaction just as in our model. In addition, the lack of the spin-orbit coupling occurs also in the description of a spin-1 particle with the gyromagnetic ratio $g=1$ moving in the external electromagnetic field [23].

\section{Acknowledgments}

This work was supported in part by a grant N 0103 U000539 from the Ministry of Education and Science of Ukraine which is gratefully acknowledged.

\section{References}

[1] L. Micu, Mod. Phys. Lett. A18, 2895 (2003).

[2] R. Tegen, R. Brockmann and W. Weise, Z. Phys. A307, 339 (1982).

[3] J. Franklin, Mod. Phys. Lett. A14, 2409 (1999).

[4] A. S. De Castro and J. Franklin, Mod. Phys. Lett. A15, 4355 (2000).

[5] M. Moshinsky and A. Szczepaniak, J. Phys. A: Math. Gen. 22, L817 (1989).

[6] D. Itó, K. Mori and E. Carriere, Nuovo Cimento A51, 419 (1967). 
[7] N. V. V. J. Swamy, Phys.Rev. 180, 1225 (1969).

[8] P. A. Cook, Lett. Nuovo Cimento 1, 419 (1971).

[9] Y. M. Cho, Nuovo Cimento A23, 550 (1974).

[10] R. Lisboa, M. Malheiro, A. S. De Castro, P. Alberto and M. Fiolhais, Phys. Rev. C69, 024319 (2004).

[11] S. Bruce and P. Mining, Nuovo Cimento A106, 711 (1993).

[12] V. V. Dvoeglazov, Nuovo Cimento A107, 1413 (1994).

[13] J. Beckers, N. Debergh and A. G. Nikitin, J. Math. Phys. 33, 3387 (1992).

[14] N. Debergh, J. Ndimubandi and D. Strivay, Z. Phys. C56, 421 (1992).

[15] V. A. Rubakov, V. P. Spiridonov, Mod. Phys. Lett A3, 1337 (1988).

[16] Y. Nedjadi and R. C. Barrett, J. Phys. A: Math. Gen. 27, 4301 (1994).

[17] M. Bednar, J. Ndimubandi and A. G. Nikitin, Can. J. Phys. 75, 283 (1997).

[18] G. Petiau, Acad. R. Belg. Cl. Sci. Mem. Collect 16, No. 2 (1936).

[19] N. Kemmer, Proc. R. Soc. 166, 227 (1938); Proc. R. Soc. A173, 91 (1939).

[20] R. J. Duffin, Phys.Rev. 54, 1114 (1939).

[21] E. M. Corson, Introduction to Tensors, Spinors, and Relativistic waveequations (Hafner Pub. Co., New York, 1953).

[22] Y. Nedjadi and R. C. Barrett, J. Math. Phys. 35, 4517 (1994).

[23] M. V. Lyubchenko and Yu. P. Stepanovsky, in Proc. Int. Conf. Heureka-2004 (Lviv) (Svit Fiziki, Lviv, 2004), p. 25. 